\title{
Plantas poco o nada conocidas de la flora vascular silvestre de las Islas Canarias
}

\author{
Arnoldo Santos-Guerra ${ }^{1}$, Jorge Alfredo Reyes-Betancort ${ }^{1}$, Miguel Antonio Padrón-Mederos ${ }^{1}$ \\ y Ricardo Mesa-Coello
}

Resumen: Santos-Guerra, A.; Reyes-Betancort, J. A.; Padrón-Mederos, M. A. \& Mesa-Coello, R. 2013. Plantas poco o nada conocidas de la flora vascular silvestre de las Islas Canarias. Bot. Complut. 37: 99-108.

\begin{abstract}
Se añaden al catálogo florístico vascular canario un total de 29 taxones. Se confirma además la presencia Epilobium angustifolium y de Chamaesyce serpens para las islas de Tenerife y La Palma respectivamente y se amplia la corología de Eleocharis palustris para la Gomera. Por otro lado reavivamos la complejidad de Sida rhombifolia L. en el Archipiélago.
\end{abstract}

Palabras clave: corología, flora, Islas Canarias, nuevas citas.

Abstract: Santos-Guerra, A.; Reyes-Betancort, J. A.; Padrón-Mederos, M. A. \& Mesa-Coello, R. 2013. Little or unknown plants of the wild vascular flora of the Canary Island. Bot. Complut. 37: 99-108.

Twenty nine new taxa are added to the checklist of the canarian vascular flora. We also confirm that Epilobium angustifolium and Chamaecyse serpens are present in Tenerife and La Palma, respectively, and the chorology of Eleocharis palustris in La Gomera is extended. On the other hand Sida rhombifolia complex is revived in the Archipelago.

Key words: chorology, Canary Islands, flora, new records.

\section{INTRODUCCIÓN}

Los catálogos florísticos intentan reflejar el conocimiento que sobre la flora de un territorio se posee en un momento puntual. Sin embargo, todos los autores encargados de poner límites al conocimiento se topan con el dinamismo del saber y por tanto con el desfase implícito de estos listados nada más ser publicados. Los nuevos estudios taxonómicos ponen en evidencia algunas de las determinaciones previas, nuevas expediciones a lugares poco 0 nada explorados, la continua introducción de nuevos elementos o el definitivo asilvestramiento de viejos conocidos trae consigo un aumento en el número de elementos silvestres a tener en cuenta; todo ello se traduce en la inherente plasticidad de todos los catálogos florísticos y en nuestro caso en particular el del Archipiélago Canario. Tomando como base el último catálogo florístico publicado para las islas (Acebes et al. 2010), aportamos en este trabajo no sólo una serie de nuevas adicio- nes a la flora silvestre de las islas Canarias, casi una treintena, sino también algunas correcciones 0 anotaciones sobre algunos taxones previamente citados con el ánimo de arrojar luz sobre su taxonomía, la confirmación de su presencia o no en las islas y la ampliación de su corología.

\section{RESULTADOS}

Presentamos los taxones por orden alfabético de familia, siguiendo la nomenclatura propuesta por los autores referenciados en los comentarios que de cada uno hacemos. Los pliegos recolectados están depositados en los herbarios del Jardín de Aclimatación de La Orotava (ORT) y la Universidad de La Laguna (TFC). Las coordenadas geográficas, cuando se indican, se corresponden con el sistema UTM (elipsoide WGS84) perteneciendo todas al huso 28, banda R (28R).

\footnotetext{
${ }^{1}$ Unidad de Botánica Aplicada (ICIA). Jardín de Aclimatación de La Orotava, C/ Retama 2, 38400 Puerto de La Cruz. Santa Cruz de Tenerife, España. areyes@icia.es

${ }_{2}$ C/ Fco. Bermúdez 6, 38500 Güímar. Santa Cruz de Tenerife, España.

Recibido: 27 febrero 2013. Aceptado: 5 abril 2013.
} 


\section{AMARANTHACEAE}

Amaranthus albus L.

La Palma: Mazo, Carretera de El Linar, 28R 0228519 3168542, $341 \mathrm{~m}$, Santos X-2003, ORT s. n.

Nativa de América del Norte, se encuentra naturalizada en Sudamérica, Europa, África del N y gran parte de Asia (Carretero in Castroviejo et al. 1990). Se ha localizado una pequeña población bien establecida en borde de carretera pero quizás desaparecida por obras de ampliación realizadas en el año 2005. En Canarias ha sido citada para las islas de Gran Canaria (Kunkel 1967) y más recientemente para Tenerife (Siverio et al. 2013). Nueva cita para la isla de La Palma.

\section{Amaranthus powelii S. Watson}

A. hybridus auct. canar. pro parte, non L.

TENERIFE: La Laguna, C/ Madre del Agua, 28R 03714763153273, 552 m, Reyes-Betancort 19-XI-2008, ORT 40636, 40637.

Especie originaria de zonas templadas de América del Norte, habiéndose naturalizado en Europa y otras partes del mundo (Carretero in Castroviejo et al. 1990). La hemos observado en comunidades nitrófilas arvenses dentro de la Vega Lagunera; muy probablemente estas plantas han sido asimiladas en el pasado a $A$. hybridus $\mathrm{L}$. s. lat. Señalada recientemente para la isla de Fuerteventura (Scholz et al. 2013). Nueva cita para la isla de Tenerife.

\section{APIACEAE \\ Ridolfia segetum Moris}

Fuerteventura: Pájara, Ajuí, cultivos abandonados, 28R 0583107 3141814, 22 m, Santos \& Fernández 24-V-1997, ORT 25370 .

Especie distribuida por el S de Europa, Anatolia, Líbano y Palestina, Azores, Canarias y N de África; naturalizada en el Perú (Aedo in Nieto-Feliner et al. 2003). Dado el escaso número de recolecciones recientes que conocemos de esta especie para Canarias parece tratarse de un elemento arvense raro, quizás en franco retroceso por el abandono de la agricultura. Citada previamente para las islas de Tenerife, Gran Canaria y Lanzarote (Acebes et al. 2010). Nueva cita para la isla de Fuerteventura.

\section{APOCYNACEAE \\ Cynanchum acutum L. subsp. acutum}

TENERIFE: Buenavista, paseo marítimo, 28R 03162703139634 , $13 \mathrm{~m}$, Santos 01-VIII-2012, ORT 46114.

Elemento presente en Asia, África y Europa (Arista \& Ortiz in Talavera et al. 2012). Hemos localizado varios ejemplares de esta subespecie junto a Patellifolia procumbens (C. Sm. ex Hornem.) A. J. Scott, Ford-Lloyd \& J. T. Williams, Chenopodium sp. y Atriplex sp., en un paseo marítimo ajardinado con Coccoloba uvifera L. Observada también en la década de los 90 en la avenida marítima de Los CristianosLas Américas (Arona-Adeje) trepando por troncos de Phoenix dactylifera L., si bien parece haber desaparecido de esta última localidad. La presencia de esta especie en las islas parece estar ligada a una introducción fortuita y casual con material de jardinería. Nueva cita para Tenerife.

\section{ASTERACEAE \\ Anacyclus clavatus (Desf.) Pers.}

TenerIFE: Buenavista del Norte, Barranco Las Cuevas Altas, 28R 0314400 3137100, 580 m, Padrón Mederos 03-V-2010, ORT 46093.

Elemento de distribución Mediterránea y Turca (Talavera in Valdés et al. 1987). Citada previamente para Lanzarote aunque sin reciente confirmación (Reyes-Betancort 1998). Este autor sugiere además la posibilidad de que probablemente se trate de una confusión con el frecuente $A$. radiatus Loisel. subsp. coronatus (Murb.) Humphries. Más recientemente, Weller (2011) recoge la presencia de una forma discoidea, subordinada a esta especie, para la isla de Fuerteventura, pero al no atribuirle paternidad alguna a esa variación no sabemos si realmente se trata de un error de determinación con el A. homogamos (Maire) Humphries (lo más factible), de un posible híbrido (A. $x$ valentinus L. 0 A. $x$ inconstans Pomel) o de una forma aún no descrita. En cualquier caso la pequeña población observada en la primavera del año 2005 no parece haber sobrevivido según datos del mismo autor. Por nuestra parte confirmamos la presencia de A. clavatus s. str. al menos en la isla de Tenerife, diferenciándose de $A$. radiatus por la presencia de brácteas involucrales internas sin ápices escariosos entre otros aspectos. Nueva cita para Tenerife.

\section{Andryala integrifolia $\mathrm{L}$.}

La Palma: Santa Cruz de La Palma, cercanías de la Montaña de Tagoja, junto a la carretera de acceso al Roque de Los Muchachos, 10- 
calmente frecuente, 28R $02278563180499,1035 \mathrm{~m}$, Santos 24-VII2011, ORT 45105.

Especie distribuida por la Región Mediterránea y el SW de Europa (Talavera in Valdés et al. 1987). Citada previamente para las islas de El Hierro y Gran Canaria (Acebes et al. 2010). Lessing (1831: 101) recoge bajo el nombre de Rothia runcinata Roth. un material recogido en la isla de Tenerife por A. Chamisso. Sobre la base de que este último taxón es sinónimo de A. integrifolia L. según el Index Kewensis, Lindinger (1926) incluye a esta especie en su listado de especies para Tenerife. Sin embargo la falta de registros actuales para la misma en esta isla nos induce a considerar la cita con precaución. Nueva cita para la isla de La Palma.

\section{Carduncellus caeruleus (L.) C. Presl.}

Carthamus caeruleus $\mathrm{L}$.

La Gomera: Alajeró, camino a Targa, Perera 05-II-2002, ORT 36747.

Elemento de distribución mediterránea y macaronésica (Valdés et al. 1987). Previamente citada para todas las islas mayores del archipiélago a excepción de El Hierro, La Gomera y Fuerteventura (Acebes et al. 2010). Scholz et al. (2013) la han citado recientemente para ésta última isla. Nueva cita para la isla de La Gomera.

Pallenis spinosa subsp. maroccana (Aurich \& Podlech) Greuter

TENERIFE: Güímar, Ladera de Güímar, camino Real, 28R 0362133 3130518, $500 \mathrm{~m}$, Reyes-Betancort \& Padrón Mederos 17-V-2011, ORT 42495, 46012; Vilaflor, 28R 0339712 3114762, 1305 m, Reyes-Betancort V-2011, ORT 46005; Adeje, Casas de Teresme, 1412 m, Ejusd. 18V-2011, ORT 42386.

Elemento del noreste africano (Marruecos y Argelia) (Aurich \& Podlech 1989). A la presencia de la subespecie tipo en Canarias (Aurich \& Podlech 1989) hemos de añadir esta segunda subespecie distinguible por sus lígulas de mayor tamaño y de color amarillo pálido. Además presenta brácteas involucrales externas más largas así como el tubo de las flores liguladas de más de $1 \mathrm{~mm}$ (Aurich \& Podlech op. cit.). Nueva cita para Canarias.

\section{Pulicaria arabica subsp. hispanica (Boiss.) Murb.}

Pulicaria paludosa Link
La Gomera: camino de Agulo a Las Rosas, 28R 02839113120347, 350 m, Reyes-Betancort \& Padrón Mederos 17-V-2011, ORT 42495.

Subespecie nativa de España, Portugal y Marruecos (Gamal-Eldin 1981). Tras una larga confusión con P. vulgaris L., ha sido recientemente reconocida por primera vez para la isla de Tenerife (Santos-Guerra \& Reyes-Betancort in Greuter \& Raab-Straube 2009). En el caso de la isla de La Gomera podría tratarse de una introducción reciente y casual dado el escaso número de ejemplares observados. Nueva cita para la isla de La Gomera.

\section{BORAGINACEAE}

Neatostemma apulum (L.) I. Johnston

LA GoMera: Alojera, Tejeleche, 28R $02717013115875,552 \mathrm{~m}$, Reyes-Betancort \& Santos 27-III-2008, ORT 41308.

Elemento de distribución general por el sur de Europa, norte de África y sudoeste de Asia (Valdés in Valdés et al. 1987). Poco frecuente, la hemos recolectado participando en comunidades de Resedo-Moricandion lanceolatae F. Casas \& M. E. Sánchez 1972. Citada previamente para las cuatro islas centro-orientales (Acebes et al. 2010). Nueva cita para La Gomera.

\section{BRASSICACEAE}

Erophila verna (L.) Chevall.

TeNERIFE: Güímar, Las Arenas, 1500 m, Mesa 01.05.2002, ORT 41062 .

Especie de amplia distribución por Europa y la región Mediterránea, SW y centro de Asia, se encuentra naturalizada en Norteamérica, Australia y Nueva Zelanda (Morales in Castroviejo et al. 1993a). Se desarrolla en prados de hierbas enanas sobre substrato de lapillis o sobre escorias recientes procedentes del Volcán de Las Arenas (erupción de 1705). Estos pequeños prados, asimilables a la alianza Tuberarion guttatae $\mathrm{Br}$.-Bl. in Br.-Bl., Molinier \& Wagner 1940 son «herbazales no nitrófilos, de desarrollo primaveral y rápido agostamiento, que colonizan suelos esqueléticos, incipientes» (Del-Arco et al. 2006). Están compuestos localmente por las siguientes especies: Arabidopsis thaliana (L.) Heynh., Aira caryophyllea L., Arenaria leptoclados (Rchb.) Guss., Galium parisiense L., Centranthus calcitrapa (L.) Dufr., Spergularia sp., Geranium molle L., Erodium sp., Vulpia myuros (L.) C. C. Gmel., Bromus madritensis L., Filago sp., Cardamine hirsuta L., Lysimachia linum-stellatum L., Myosotis disco- 
lor subsp. canariensis Grau y Senecio vulgaris L. El taxón en cuestión parece estar confinado a una pequeña área en la base suroccidental del volcán de Las Arenas, donde se le viene observando desde la década de los 70 del pasado siglo, sin que muestre tendencia a ampliar su areal de distribución. Por la proximidad a un sendero muy transitado por excursionistas y peregrinos es muy probable que esta pequeña población tenga su origen en una introducción fortuita. Nueva cita para Canarias.

\section{CISTACEAE}

Cistus ladanifer f. maculatus (Dunal) Samp.

TENERIFE. Tenerife, Los Realejos, zona alta de Las Llanadas, Delgado \& Hernández 08-VI-2009, ORT 41426.

Taxón distribuido de modo natural por la Península Ibérica y sur de Francia (Demoly \& Montserrat in Castroviejo et al. 1993b). En Tenerife aparece asilvestrada localmente producto probablemente de una introducción accidental con pinos de repoblación. Introducida previamente en la isla de Gran Canaria (Sunding \& Kunkel 1966). Nueva cita para la isla de Tenerife.

\section{CONVOLVULACEAE \\ Cuscuta campestris Yunck}

TeNeRIFE: La Laguna, bordes de charcas entre Tejina y Bajamar, 28R 03672473158946,121 m, Herrera García s.d., ORT 36952.

Originaria de Norteamérica, se halla naturalizada en todo el mundo. Ha sido introducida fundamentalmente como parásita de la alfalfa y otras leguminosas forrajeras (García in Talavera et al. 2012). En Tenerife ha sido observada de manera puntual ligada a cañaverales de Arundo donax L. Nueva cita para Canarias.

\section{CRASSULACEAE}

\section{Aeonium palmense Webb ex Christ}

A. canariense subsp. christii (Burchard) Bañares; A. canariense var. palmense (Webb ex Christ) H.Y. Liu

Tenerife: Buenavista, Masca, 28R $03195943132678,617 \mathrm{~m}$, Santos 1999, ORT 35218, 35219.

Endemismo hasta ahora considerado como exclusivo de la isla de La Palma (Liu 1989, Acebes et al. 2010). Nosotros lo hemos observado de manera frecuente en el sector occidental seco del macizo de Teno, en comunidades rupícolas de los barrancos de Carrizales, Juan López, Masca, El Natero y Seco junto a las especies Phyllis viscosa Webb ex Christ y Aeonium sedifolium (Webb ex Bo1le) Pit. \& Proust, entre otras. Las citas de A. canariense s. str. para este sector de la isla de Tenerife deben referirse a A. palmense. Estudios más profundos son necesarios para saber más sobre las relaciones florísticas ya ejemplarizadas con otra especie de Aeonium (A. sedifolium) entre el oeste de Tenerife y la isla de La Palma. Nueva cita para la isla de Tenerife.

\section{CUCURBITACEAE \\ Momordica charantia L.}

La Palma: San Andrés y Sauces, San Andrés, Bco. del Agua, 28R 02305653189065,5 m, Santos 30-IX-2012, ORT 45262; Ibid., Santos \& Fernández 21-VIII-2012, ORT 46113.

Elemento Pantropical; posiblemente introducido en el Nuevo Mundo (Jeffrey 1978). Recolectada en lugares nitrofilizados cerca del cauce del barranco de Agua. Por el pequeño tamaño del fruto nuestro material sería asimilable a la var. muricata (Willd.) Chakrav. considerada como la forma silvestre del taxón cultivado (var. charantia) (Joseph \& Antony 2010). Ya Viera y Clavijo (1866) recoge en su Diccionario de Historia Natural, bajo la voz «Balsamina», su presencia como cultivada «en algunos huertos y casas de nuestras islas» y de la que dice «La balsamina es vulneraria, y anodina; puesta en aceite al sol pasa por un excelente bálsamo para las hemorroides, picaduras de tendones, y pelos en los pechos de las que crían». Nueva cita para Canarias.

\section{CYPERACEAE}

Bolboschoenus glaucus (Lam.) S. G. Smith

Bolboschoenus maritimus sensu auct. non (L.) Pallas

Tenerife: La Laguna, Tejina, charcas artificiales cerca del enlace norte al casco, 28R $03673513158138,115 \mathrm{~m}$, Santos 5-X-2010, ORT 45433; Ibid., charca de Tejina, Pérez de Paz 28-VII-1985, TFC 24459; Ibid., Ejusd. VII-1985, TFC 24628.

Esta especie se distribuye por África, próximo Oriente, la India y $\mathrm{C}$ y $\mathrm{S}$ de Europa, hallándose introducida en Norteamérica (Martín Bravo et al. in Castroviejo et al. 2008). Según estos autores ha existido una confusión imperante en la concepción de Bolboschoenus maritimus s. str., lo que nos sugiere la conveniencia de revisar el mate- 
rial canario asignado a esta especie y confirmar que se trate realmente de este taxón y no del aquí mencionado $B$. glaucus. Por nuestra parte hemos revisado los pliegos depositados en el herbario del Departamento de Biología Vegetal de la Universidad de La Laguna (TFC) y hemos confirmado que se trata de este último taxón y no de $B$. maritimus. Nueva cita para Canarias.

Eleocharis palustris (L.) Roem. \& Schult. subsp. palustris

LA GoMera: Valle Gran Rey, salida de Arure para Las Hayas, 28R $02728643113212,837 \mathrm{~m}$, Santos 02-VII-2003, ORT 37194.

Elemento propio de Eurasia y el N de África (Jiménez Mejías \& Luceño in Castroviejo et al. 2008). Para La Gomera conocíamos únicamente la cita de Barranco de Agando sobre Benchijigua 800 m (Lid 1967), observándola nosotros también, si bien de manera escasa, en una pequeña represa a la salida de Arure, junto a Ruppia maritima L., Scirpoides holoschoenus subsp. globiferus (L. f.) Soják y Mentha sp., entre otras. Esta especie ha sido citada además para las islas de Tenerife y Gran Canaria (Acebes et al. 2010). Aportación corológica para la isla de La Gomera.

\section{EUPHORBIACEAE}

Chamaecyse hypericifolia (L.) Millsp.

Euphorbia hypericifolia L.

LA GoMera: Valle Gran Rey, jardines entrada Hotel Gran Rey, 2 m, Padrón Mederos, Reyes-Betancort \& Guma 28-VI-2010, ORT 42468.

Especie que se halla distribuida desde Venezuela y Colombia hasta Georgia, Florida y Texas (EE.UU.) (Correll \& Johnston 1970). Posiblemente de reciente introducción, se ha recolectado en ambientes antropizados como bordes de carreteras, parterres, etc., junto a Portulaca nicaraguensis (Danin \& H. G. Baker) Danin. Citada previamente para las islas de El Hierro, Tenerife, Gran Canaria y Fuerteventura (Padrón-Mederos et al. 2007). Nueva cita para la isla de La Gomera.

\section{Chamaesyce serpens (Kunth) Small}

LA PALMA: Breña Alta, Los Guinchos, comunidades herbáceas halo-nitrófilas, 28R 02296783173827,7 m, Santos 10-X-2010, ORT 45424 .
Originaria de América tropical, se ha naturalizado ampliamente en buena parte de las regiones templadas (Benedí in Castroviejo et al. 1997). Posiblemente introducida con sustratos y semillas de uso en jardinería. Citada previamente para la isla de La Palma en la localidad de Puerto Naos (Santos-Guerra 1996), parece que este hallazgo ha pasado desapercibido para Acebes et al. (2010). Confirmamos y ampliamos aquí su distribución hacia el Este palmero.

\section{Phyllanthus tenellus Roxb.}

La Palma: Breña Baja, Los Cancajos, en bordes de aceras, 28R 0230236 3171840, 27 m, Santos 10-X-2010, ORT 45418

Especie pantropical (Silva \& Sales 2008), se comporta como mala hierba en huertos y jardines siendo aún una planta rara en la isla. Probablemente la cita de P. niruri L. para Gran Canaria (Kunkel 1971) se ha de referir a esta especie. Recientemente Padrón-Mederos et al. (2009) la han confirmado también para la isla de Tenerife. Nueva cita para la isla de La Palma.

\section{FABACEAE \\ Lotus edulis L.}

La Palma: Mazo, jardines desaparecidos del aeropuerto, 28R 02308233169085 , 29 m, Santos 10-IV-2005, ORT s.n.; Ibid., Ejusd. 17-IV-2005, ORT 39146.

Especie nativa del S de Europa, SW de Asia y N de África (Valdés in Talavera et al. 2000). Elemento casual en nuestra flora, introducido probablemente con sustratos y material vegetal de jardinería o maquinaria en la construcción del aeropuerto. Nueva cita para Canarias.

\section{Medicago lupulina L.}

Lanzarote: Arrecife, rotonda a la salida hacia Tahiche, en céspedes, 28R 0641731 3206390, Reyes-Betancort 20-VII-2011, ORT 46019.

Especie presente en Europa, N y E de África, SW y C de Asia y Macaronesia además de naturalizada en Australia, Japón, $\mathrm{N}$ de América y S de África (Sales \& Hedge in Talavera et al. 2000). En Canarias parece hallarse localizado en ambientes bastantes antropizados, tales como borde de vías, parterres, jardines, etc. Citada previamente pa- 
ra las islas de El Hierro, La Palma y Tenerife (Acebes et al. 2010). Nueva cita para la isla de Lanzarote.

\section{Trifolium cernuum Brot.}

Tenerife: El Tanque, al oeste de Ruigómez, 28R 0324044 3136787, 787 m, Padrón-Mederos 08-V-2011, ORT 46085.

Taxón nativo del SW, C y E de Europa, SW de Asia y NW de África (Muñoz Rodríguez et al. in Talavera et al. 2000). Hemos observado pocos ejemplares en pistas agrícolas del NW de la isla de Tenerife, lo que sugiere una posible introducción con motivo de las labores de plantación y abonado de las fincas adyacentes. Citada con anterioridad para Canarias, en concreto para la isla de El Hierro, por Stierstorfer \& v. Gaisberg (2006). Nueva cita para Tenerife.

\section{Trifolium repens $\mathrm{L}$.}

Tenerife: El Tanque, al oeste de Ruigómez, 28R 0323855 $3136381,826 \mathrm{~m}$, Reyes-Betancort, Santos \& Padrón-Mederos 06-VII2011, ORT 46086; La Orotava, subida Aguamansa por los Bebederos, 28R 0352789 3139173, 901 m, Padrón-Mederos 10-V-2011, ORT 46087.

Elemento distribuido por debajo del meridiano $70^{\circ} \mathrm{N}$ en Europa, regiones mediterránea y macaronésica; estando introducido en áreas muy diversas: Norteamérica, $\mathrm{S}$ de África, islas del Atlántico, Australia y E de Asia (Muñoz Rodríguez et al. in Talavera et al. 2000). En Canarias es frecuente en céspedes artificiales, sin embargo también ha sido observado asilvestrado en bordes de caminos rurales. Según Acebes et al. (2010) ha sido citado para las islas de El Hierro, Gran Canaria y Fuerteventura. Nueva cita para la isla de Tenerife.

\section{LILIACEAE Allium pallens L.}

LANZAROTE: Haría, La Montaña, 28R 06444643222059,578 m, Reyes-Betancort 18-VII-2011, ORT 46020; Haría, Valle de Temisa, Ejusd. (!).

Especie restringida al Mediterráneo occidental y central, alcanzando Grecia por el este (Pastor \& Valdés 1982). Este taxón pertenece a la sección Codonoprasum Rchb. existiendo cierta controversia en la delimitación de los taxones integrantes en la misma (Aedo 2012). Para su identificación hemos seguido a Brullo et al. (2008) los cuales consideran que Allium pallens difiere de A. dentiferum Webb \& Berthel. por poseer la túnica externa de los bulbos blanquecina, una inflorescencia muy compacta y globosa, tépalos blancos cortos, estambres exertos sin dientes interestaminales y ovario liso. Las diferencias entre esta definición y la propuesta para A. stearnii Pastor \& Valdés por Aedo (2012) hacen difícil su separación, hecho quizás observado también por Govaerts (2012) quién los considera como meros sinónimos. La hemos visto asilvestrada en terrazas de cultivo abandonadas, así como en bordes de cultivos en la zona alta del municipio de Haría. Nueva para Canarias.

\section{MALVACEAE \\ Malvastrum corchorifolium (Desr. in Lam.) Britton ex Small}

La Palma: Barlovento, carretera de acceso a Puerto de Talavera, 28R 0229201 3192250, c. 35 m, Santos 4-II-2012, ORT 45460; San Andrés y Sauces, acceso a San Andrés por el sur, 28R 0230323 3188268, 108 m, Ejusd. II-2013, ORT 45859.

Se halla distribuido por Florida, S de México hasta Nicaragua e islas del Caribe e introducida en Cabo Verde y Ghana (Hill 1982). Según este mismo autor parece ser un híbrido entre M. americanum (L.) Torrey y M. coromandelianum (L.) Garcke. Se distingue del otro representante del género presente en las islas, M. coromandelianum subsp. coromandelianum (subespecie confirmada por nosotros para Gran Canaria por ejemplo en Arucas y Las Palmas de Gran Canaria), por el escaso desarrollo de las 3 espinas (meras protuberancias) que adornan la parte superior de los mericarpos, el indumento doble de su cáliz y por sus inflorescencias terminales a modo de cortas espigas. En La Palma la hemos recolectado en bordes de carretera en comunidades herbáceo-arbustivas nitrófilas, siendo localmente frecuente. Citada previamente para la isla de Gran Canaria (San Lorenzo) por Kunkel (1968). Nueva cita para la isla de La Palma.

\section{Sida rhombifolia var. canariensis (Willd.) Griseb}

LA PALMa: San Andrés y Sauces, bajada a San Andrés (sur), 28R 0230298 3188274, 110 m, Santos 4-XI-2012, ORT 45725; Los Llanos de Aridane, Barrio del Mensajero, Cabrera Rguez. III-1989, TFC 25759. Tenerife: Los Silos, Barranco del Agua, 200 m, Svent. 26-VI-1949, ORT 2802, 9013, 16693, 16694; Ibid., hacia Barranco del Agua, Ejusd. 20-IV-1963, ORT 16690; Ibid., La Isleta, Lucía Sauquillo \& Reyes-Betancort 29-VI-1997, TFC 40835; prope Hortum Orotava, ad vias, Burchard IX-1911, ORT 328; El Sauzal, canal de riego, Barquín \& del Ar- 


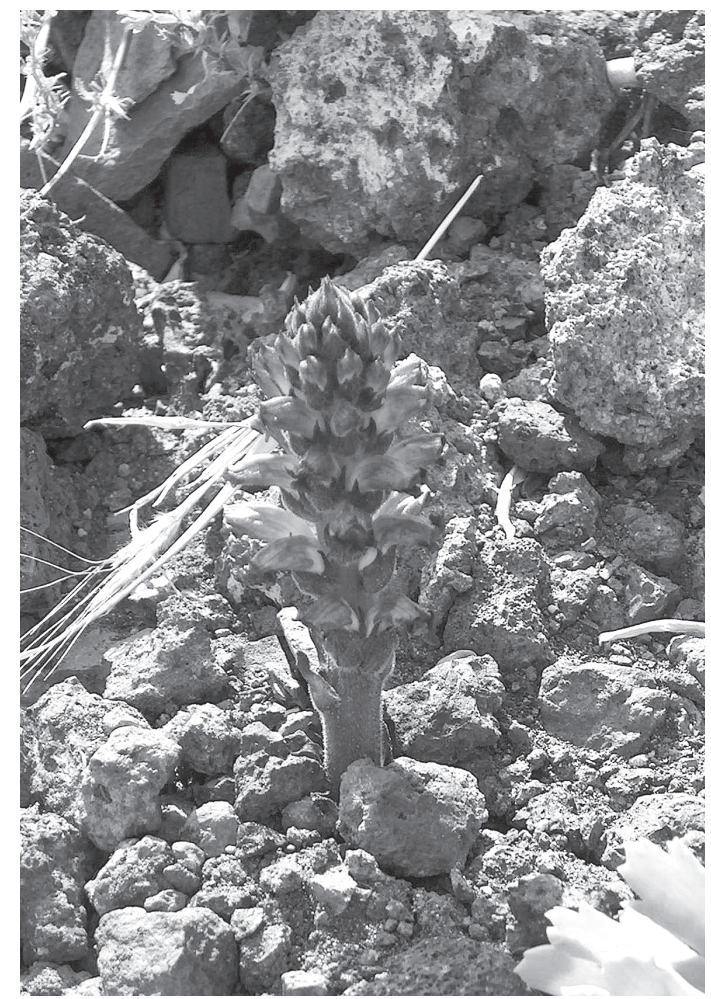

Fig. 1- Orobanche cernua var. desertorum. Costa de Alojera, 20-IV-2005 (foto R. Mesa).

co 27-VIII-1975, TFC 22974; Tacoronte, Barranco de Guayonje, 28R 0360964 3152169, 365 m, Rguez. Navarro 16-XI-2008, TFC 48969; La Laguna, Punta del Hidalgo, La Serna 02-VI-1973, TFC 35748; Santa Cruz de Tenerife, Barranco Igueste, Svent. 27-II-1944, ORT 16692; Arona, Wildpret \& Acebes 02-III-1975, TFC 4513. Gran Canaria: Guía, Ingenio Blanco, Barranco Las Hayas, Suárez Rguez. 03-XI-1985, TFC 31226.

var. maderensis (Lowe) Lowe

La Palma: Los Sauces, Sventenius 22-X-1945, ORT 4291. La GoMera: Taguluche, 400 m, Sventenius 13-V-1959, ORT 6422; La Laja, 400 m, Ejusd. 29-III-1959, ORT 6423; Barranco Caviño 200 m, Ejusd. 15-V-1945, ORT 6424; Taguluche del Sur, 28R 0270310 3114371, 130 m, Padrón Mederos 02-X-2005, TFC 47181. TeNERIFE: Santa Cruz de Tenerife, Barranco del Bosque [iBrosque?] (Anaga), 250 m, Barquin 06-XII-1975, TFC 4812.

A falta de trabajos más profundos en lo que concierne a la variabilidad de esta especie en su amplio rango de distribución reconocemos por nuestra parte dos variedades en las islas Canarias. Por un lado, S. rhombifolia var. canariensis, distribuida en las islas de La Palma, Tenerife y Gran Canaria, la cual se diferenciaría por la presen- cia de dos aristas en el ápice de los mericarpos, siendo éstas más cortas que en la variedad tipo (Schumann 1891), y por otro lado S. rhombifolia var. maderensis que se encuentra presente en las islas de La Palma, La Gomera y Tenerife (véase también Lowe 1868: 69-70, 592). Esta variedad, ampliamente distribuida por los trópicos (Verdcourt 2004), se caracteriza principalmente por la presencia de una sola arista en el ápice de los mericarpos (Lowe 1868; Verdcourt op. cit.) y por el menor tamaño de éstos (c. $2,5 \mathrm{~mm}$ excl. arista frente a los $3 \mathrm{~mm}$ que alcanzan en la var. canariensis). Confirmación para Canarias.

\section{OENOTHERACEAE \\ Epilobium angustifolium $\mathrm{L}$.}

Chamaenerion angustifolium (L.) Scop.

TenerIFE: La Orotava, Aguamansa, cerca de Los Órganos, 28R 0353334 3136627, 1374 m, Santos 06-IV-2004, ORT 37943; ex horto Jard. Aclimatación Orotava (plantas provenientes de Aguamansa), Ejusd. V-2004, ORT 45675.

Zonas templadas y frías del Hemisferio Norte (Nieto Feliner in Castroviejo et al. 1997). La especie no había vuelto a ser citada desde que Bolle (1861) la mencionara en su descripción de la zona alta y desierta de Tenerife junto a Sorbus aria (L.) Crantz, Arabis albida (= Arabis alpina subsp. cancasica (Willd.) Briq.), Bromus tectorum L. y Rosa canina L. Nosotros la hemos observado como muy rara en lugares húmedos de áreas de pinar, cerca de Los Órganos con Rosa canina L., Bystropogon canariensis var. smithianus Christ y Sideritis oroteneriffae Negrín $\&$ P. Pérez. Confirmación para Canarias.

\section{OROBANCHACEAE}

\section{Orobanche cernua var. desertorum Beck (Fig. 1)}

Tenerife: Granadilla, proximidades del Polígono Industrial, 28R 0351903 3108171, 75 m, Mesa 04-III-2011, ORT 46027; Arico, Los Abades, Pérez de Paz 19-II-1996, TFC 29704. La Gomera: Vallehermoso, costa de Alojera, sendero a Galión, Mesa 20.IV.2005 (!).

Esta especie ha sido citada con anterioridad para Lanzarote y Gran Canaria (Uhlich 1994), El Hierro (Wolf \& Rosinski 1999) y para Fuerteventura (Scholz et al. 2004). Especie distribuida por el norte de África hasta la India, posiblemente nativa en Canarias. Resulta curioso lo extremadamente escaso que es este taxón en Tenerife, donde solamente se han observado contados ejemplares en el sur de la isla, siempre en años muy lluviosos y siempre en las proximidades de zonas de encharcamiento. En esta isla 
posiblemente parasite a Schizogyne sericea (L.f.) DC. en matorral de sustitución dominado por esta especie acompañado por Argyranthemum frutescens subsp. gracilescens (Christ) Humphries, Polycarpaea nivea (Ait.) Webb, Frankenia ericifolia C. Sm. ex DC. subsp. ericifolia, Nicotiana glauca Graham y Caesalpinia spinosa (Molina) Kuntze. En La Gomera solamente se ha observado en la costa de Alojera, en matorral costero con Astydamia latifolia (L.f.) Baill. Por el contrario, la especie resulta ser frecuente en El Hierro donde parasita a Schizogyne sericea (Wolf \& Rosinski op. cit.) así como en las islas orientales. Nueva cita para las islas de La Gomera y Tenerife.

\section{POACEAE}

\section{Catapodium rigidum subsp. hemipoa}

(Delile ex Spreng.) Kérguelen

Lanzarote: Haría, Montaña Aganada, Santos 03-IV-1996, ORT 32435 .

Elemento del oeste de la Región Mediterránea (Devesa in Valdés et al. 1987). Las únicas citas publicadas para las islas Canarias se deben a Marrero et al. (1995) quienes la localizan en los parajes afectados por las erupciones históricas de Timanfaya. Nosotros hemos observado esta subespecie en el Norte de la isla, más concretamente en el macizo de Famara. Llama la atención la particular ecología de esta especie en la isla de Lanzarote, al ausentarse de los arenales marítimos o de interior a los que se asocian al menos en el Norte de África y la Península Ibérica (Maire 1955, Devesa in Valdés et al. 1987). Nueva 10calidad de esta subespecie poco recolectada.

\section{Poa trivialis L.}

La PaLma: Puntallana, Cubo de La Galga, 28R 02278193183641, 804 m, Santos 24-IV-1997, ORT 34376.

Este taxón se encuentra diseminado por Europa, $\mathrm{N}$ de África, Asia y Macaronesia hallándose introducida en otras partes del Globo (Devesa in Valdés et al. 1987). Citada con anterioridad para las islas de Tenerife y Lanzarote (Acebes et al. 2010). Nueva para la isla de La Palma.

\section{PSILOTACEAE}

Psilotum nudum (L.) P. Beauv.

TENERIFE: La Laguna, subespontánea en los jardines del Instituto Canario de Investigaciones Agrarias (Valle Guerra), 28R 0364318
3154463, 342 m, Santos 5-VII-2011, ORT 45139; Puerto de La Cruz, jardines y anexos del Jardín de Aclimatación de la Orotava, Santos (!). La Palma: Santa Cruz de La Palma, Jardines particulares de Antonio Galván, Santos (!).

Se halla distribuida por regiones intertropicales, Macaronesia (Cabo Verde) y S de la Península Ibérica (Castroviejo in Castroviejo et al. 1986). Observada tanto como epífita, sobre roca 0 en suelo, probablemente introducida con elementos de jardinería.

Nueva para Canarias.

\section{SOLANACEAE}

Datura ferox $\mathrm{L}$.

Tenerife: Arafo, Volcán de Arafo, Finca El Carretón, 28R $03640913134520,130 \mathrm{~m}$, Castro \& Massanet 04.2011, ORT s.n.

Según Gallego in Talavera et al. (2012) es una especie procedente de Asia y naturalizada en América y en la región Mediterránea. Por nuestra parte la hemos observado en Tenerife como mala hierba de carácter puntual en cultivos, posiblemente de reciente introducción. Nueva cita para Canarias.

\section{VALERIANACEAE \\ Valerianella microcarpa Loisel.}

TenerIFE: Santiago del Teide, Teno, Barranco Seco, 28R 0320401 $3129047,470 \mathrm{~m}$, Reves-Betancort \& Padrón Mederos 07-III-2010, ORT 41484. LA Gomera: Alojera, camino hacia Tejeleche, 28R 0271382 3116135, 540 m, Reyes-Betancort et al. 19-III-2010, ORT 41473.

Especie presente en la Región Mediterránea, Madeira y E de África (Devesa \& López Martínez in Devesa et al. 2007). Observada formando parte de pastos de corta talla en senderos y caminos, pertenecientes a la alianza Tuberarion guttatae. Nueva cita para Canarias.

\section{AGRADECIMIENTOS}

Al Prof. Juan Ramón Acebes Ginovés, curator del herbario de la Universidad de La Laguna que como siempre nos permitió amablemente la consulta del material depositado allí y al que hacemos referencia en este trabajo. Al Dr. Filip Verloove, del Jardín Botánico Nacional de Bélgica, quién también amablemente nos facilitó bibliografía de interés. A D. Luis Delgado y a D. Miguel Ángel Hernández de la Unidad de Flora del Centro Ambiental La Tahonilla, a D. Cecilio Massanet y D. Raúl Herrera del Instituto Canario de Investigaciones Agrarias, por hacernos llegar material de estas nuevas citas. 


\section{BIBLIOGRAFÍA}

Acebes GinOvés, J. R.; LeÓn ARENCIBIA, M. C.; RodríGUEZ-NAvarro, M. L.; Del Arco Aguilar, M.; García Gallo, A.; PéreZ de Paz, P. L.; Rodríguez Delgado, O.; Martín OsoRio, V. E. \& WildPret de LA TORRe, W. 2010. Pteridophyta, Spermatophyta. En: M. Arechavaleta, S. Rodríguez, N. Zurita \& A. García (Eds.), Lista de especies silvestres de Canarias (hongos, plantas y animales terrestres). 2009: 119-172. Gobierno de Canarias.

AEdo, C. 2012. Allium L. En S. Castroviejo et al. (Eds.), Flora Iberica, 20: http://www.floraiberica.es/floraiberica/texto/ borradores/vol_XX/20_183_27_Allium.pdf[Acceso junio 2012].

Aurich, CH. \& Podlech, D. 1989. Zur gliederung von Asteriscus spinosus (L.) Schultz-Bip. sensu lato. (Compositae). Mitt. Bot. Staatssamml. München 28: 239-296.

Bolle, C. 1861. Die Canarischen Inseln. I. Z. Allg. Erdk. 10: $1-32$.

Brullo, S.; Guglielmo, A.; Pavone, P. \& Salmeri, C. 2008. Taxonomic study on Allium dentiferum Webb \& Berthel. (Alliaceae) and its relations with allied species from the Mediterranean. Taxon 57(1): 243-253.

Castroviejo, S.; Aedo, C.; Benedí, C.; Laínz, M.; Muñoz Garmendia, F.; Nieto Feliner, G. \& Pavia, J. (Eds.) 1997. Flora Iberica, 8. Real Jardín Botánico, Madrid.

Castroviejo, S.; Aedo, C.; Gómez Campo, C.; Laínz, M.; Montserrat, P.; Morales, R.; MuÑoz Garmendia, F.; Nieto Feliner, G.; Rico, E.; Talavera, S. \& Villar, L. (Eds.) 1993a. Flora Iberica, 4. Real Jardín Botánico, CSIC, Madrid.

Castroviejo, S.; Aedo, C.; Cirujano, S.; Laínz, M.; Montserrat, P.; Morales, R.; MuÑoz Garmendia, F.; Navarro, C.; Paiva, J. \& Soriano, C. (Eds.) 1993b. Flora Iberica, 3. Real Jardín Botánico, Madrid.

Castroviejo, S.; Laínz, M.; LóPez GonzÁlez, G.; Montserrat, P.; Muñoz Garmendia, F.; Paiva, J. \& Villar, L. (Eds.) 1986. Flora Iberica, 1. Real Jardín Botánico, CSIC, Madrid.

Castroviejo, S.; Laínz, M.; López GonzÁlez, G.; Montserrat, P.; MuÑoz Garmendia, F.; Paiva, J. \& Villar, L. (Eds.) 1990. Flora Iberica 2. Real Jardín Botánico, CSIC, Madrid.

Castroviejo, S.; Luceño, M.; Galán, A.; Jiménez Mejías, P.; Cabezas, F. \& Medina, L. (Eds.) 2008. Flora Iberica, 18. Real Jardín Botánico, CSIC, Madrid.

Correll, D. S. \& Johnston, M. C. 1970. Manual of the vascular plants of Texas. Texas Research Foundation, Renner, Texas.

del-Arco, M. J.; Wildpret, W.; PéreZ-De-Paz, P. L.; RodríGuez, O.; Acebes, J. R.; García Gallo, A.; Martín, V. E.; Reyes-Betancort, J. A.; Salas, M.; Díaz, M. A.; Bermejo, J.A.; González GonzÁlez, R.; Cabrera, M. V. \& GarCía Ávila, S. 2006. Mapa de Vegetación de Canarias. GRAFCAN Ediciones, Arafo.

Devesa, J. A.; Gonzalo, R. \& Herrero, A. (Eds.) 2007. Flora Iberica 15. Real Jardín Botánico, CSIC, Madrid.
Gamal-Eldin, E. 1981. Revision der Gattung Pulicaria (Compositae-Inuleae) für Afrika, Makaronesien und Arabien. J. Cramer, Vaduz.

GovaERTS, R. 2012. World checklist of selected plant families. Royal Botanic Gardens, Kew. http://apps.kew.org/wcsp/ synonomy.do?name_id=296184 [Acceso Octubre 2012]

Greuter, W. \& RaAb-StRaube, E. V. 2009. Euro+Med notulae, 4. Willdenowia 39: 327-333.

HiLl, S. R. 1982. A monograph of the genus Malvastrum A. Gray (Malvaceae: Malveae). Rhodora 84: 1-83; 159-264; 317-409.

Jefrerey, C. 1978. Cucurbitaceae. En E. Launert (Ed.), Flora Zambesiaca, 4: 414-499. Flora Zambesiaca Managing Committee, London, United Kingdom.

JoSEPH, J. K. \& ANTONY, V. T. 2010. A taxonomic revision of the genus Momordica L. (Cucurbitaceae) in India. Indian J. Plant Genet. Resour. 23(2): 172-184.

Kunkel, G. 1968. Nuevas plantas para la flora canaria. Cuad. Bot. Canaria 3: 57-58.

KunKEL, G. 1971. Notas sobre algunas plantas asilvestradas en Gran Canaria. Cuad. Bot. Canaria 11: 1-3.

LESSING, C. F. 1831. De plantis in expeditione speculatoria romanzoffiana observatis disserere: Synanthereae Rich. Linnaea 6: 83-170.

LID, J. 1967. Contributions to the flora of the Canary Islands. Skr. Norske. Vidensk.-Akad. I. Oslo. Mat.-Naturv. Kl. 23: $1-212$.

Lindinger, L. 1926. Beiträge zur Kenntius von Vegetation und Flora der Kanarischen Inseln. Abh. Auslandsk., Reihe C, Naturwiss. 21: 1-350.

Liu, H. Y. 1989. Systematics of Aeonium (Crassulaceae). Taichung (Taiwan). Natl. Mus. Nat. Sci. Special Publ. 3.

Lowe, R. T. 1868. A manual for flora of Madeira and the adjacent Islands of Porto Santo and the Deserts, 1. John Van Voorst, London.

Maire, R. 1955. Flore de l'Afrique du Nord, 3. Paul Lechevalier Ed. París.

Marrero, A.; GonzÁlez-Martín, M.; Betancort Villalba, M. J.; Carrasco, A. \& Perdomo, A. 1995. Adiciones y comentarios sobre la flora vascular de Lanzarote. Bot. Macaronésica 22: 91-110.

Nieto Feliner, G.; Jury, S. L. \& Herrero, A. 2003. Flora Iberica, 10. Real Jardín Botánico, CSIC, Madrid.

Padrón-Mederos, M. A.; León Arencibia, M. C. \& Reyes-BeTANCORT, J. A. 2009. Novedades corológicas y comentarios para la flora vascular de El Hierro y La Palma (islas Canarias). Vieraea 38: 1-16.

Padrón-Mederos, M. A.; Reyes-Betancort, J. A.; GonzÁlez GonZÁlez, R. \& LeÓn Arencibia, M. C. 2007. Adiciones y comentarios a la flora vascular de Canarias. Vieraea 35 : 43-50.

Pastor, J. \& Valdés, B. 1982. Revisión del género Allium (Liliaceae) en la Península Ibérica e Islas Baleares. Anal. Univ. Hispalense, Ser. Ci., Otras Publ. Sevilla. 
Reyes-BetANCORT, J. A. 1998. Flora y Vegetación de la isla de Lanzarote (Reserva de la Biosfera). Departamento de Biología Vegetal, Universidad de La Laguna. Tesis Doctoral (Inéd.).

Rivas-MarTínez, S.; FERnÁNDEZ-GonZÁLEZ, F.; LoIdi, J.; LouSA, M. \& PENAS, A. 2001. Syntaxonomical checklist of vascular plant communities of Spain and Portugal to association level. Itin. Geobot. 14: 5-341.

Rivas-Martínez, S.; Díaz, T. E.; Fernández-GonzÁlez, F.; IzCo, J.; LoIdi, J.; LousA, L. \& PENAS, A. 2002. Vascular plant communities of Spain and Portugal. Addenda to the syntaxonomical checklist of 2001. Itin. Geobot. 15(1-2): 1-922.

RodrígueZ Delgado, 0.; DEL-Arco Aguilar, M. J.; García GALlo, A.; Acebes Ginovés, J. R.; PÉrez De PAz, P. L. \& WildPRET DE LA TORRE, W. 1998. Catálogo sintaxonómico de las comunidades vegetales de plantas vasculares de la Subregión Canaria: Islas Canarias e Islas Salvajes. Colección Materiales Didácticos Universitarios 2, serie Biología 1. Servicio de Publicaciones de La Universidad de La Laguna.

SANTOS-GuERrA, A. 1996. Notas corológicas III: adiciones florísticas y nuevas localidades para la flora canaria. Anales Jard. Bot. Madrid 54: 445-448.

Scholz, S.; Reyes-Betancort, J. A.; Scholz, H. \& WildPret, W. 2004. Adiciones a la flora vascular de Fuerteventura (Islas Canarias). Bot. Macaronésica 25: 165-174.

Scholz, S.; Reyes-Betancort, J. A. \& WildPREt, W. 2013. Adiciones a la flora vascular de Fuerteventura (Islas Canarias). III. Bot. Macaronésica 28, 2010 (en prensa).

Schumann, K. 1891. Sida L. En K. F. P. von Martius (Ed.) Flora Brasiliensis, 12(3): 279-347.

Silva, M. J. DA \& SALES, M. F. 2008. Sinopse do gênero Phyllanthus (Phyllanthaceae) no nordeste do Brasil. Rodriguézia 59(2): 407-422.
Siverio-NúÑez, A.; Sobrino, E.; Rodríguez de la Torre, H. A.; Reyes-Betancort, J. A. \& Santos-Guerra, A. 2013. Nuevos xenófitos de elevada capacidad invasora para la flora canaria. Bot. Macaronésica 28, 2010 (en prensa).

Stierstorfer, C. \& von Gaisberg, M. 2006. Annoted checklist and distribution of the vascular plants of El Hierro, Canry Islands, Spain. Englera 27: 1-221.

Talavera, S.; Aedo, C.; Castroviejo, S.; Herrero, A.; Romero Zarco, C.; Salgueiro, F. J. \& Velayos, M. 2000. Flora Iberica, 7(2). Real Jardín Botánico, Madrid.

Talavera, S.; Andrés, C.; Arista, M.; Fernández Piedra, M. P.; Gallego, M. J.; Ortiz, P. L.; Romero Zarco, C.; SALGueiro, F. J.; Silvestre, S. \& Quintanar, A. 2012. Flora Iberica, 11. Real Jardín Botánico, CSIC, Madrid.

UhLich, H. 1994. Ein Beitrag zur Kenntnis von Orobanche berthelotii Webb et Berthelot. Gleditschia 22(1): 23-24.

Valdés González, B.; Talavera, S. \& Fernández Galiano, E. (Eds.) 1987. Flora vascular de Andalucia occidental. Ketres Editora S. A. Barcelona.

VERdCOURT, B. 2005. The variation of Sida rhombifolia L. (Malvaceae) in East Africa. Kew Bull. 59: 233-239.

Viera y Clavijo, J. 1866. Diccionario de historia natural de las Islas Canarias, ó Índice alfabético descriptivo de sus tres reinos animal, vegetal y mineral, I. Real Sociedad Económica de Amigos del País de Gran Canaria. Imp. de la Verdad, Las Palmas de Gran Canaria.

WeLLER, A. A. 2011. New records and noteworthy range extensions of vascular plants from the Canary Islands and Madeira. Bocagiana 234: 1-11.

WolfF, P. \& Rosinski, M. 1999. Nuevas e interesantes plantas vasculares en las islas Canarias. Vieraea 27: 11-21. 